\title{
THE HEMODYNAMIC EFFECTS OF ANGIOTONIN IN NORMAL MAN ${ }^{1}$
}

\author{
By STANLEY E. BRADLEY AND BARBARA PARKER \\ (From the Departments of Medicine and Physiology, New York University College of Medi- \\ cine, and the Third (New York University) Medical Division of Bellevue Hospital, \\ New York City)
}

(Received for publication July 16, 1941)

Angiotonin, a pressor substance arising out of the reaction between renin and renin-activator, has been suggested by Page and Helmer (1) as the humoral mediator of clinical and experimental hypertension. The vasopressor activity of this substance in animals is not abolished by anesthesia or pithing; it causes vasoconstriction in the isolated rabbit's ear (1) and reduces the renal blood flow by constriction of the efferent glomerular arterioles (2), facts which lead to the belief that it acts directly on the peripheral vascular bed. It is also known that it decreases the diastolic volume in the isolated mammalian heart (3). In man, the effects are similar to those occurring in animals, namely, hypertension without change in skin temperature, and diminished renal blood flow occasioned by efferent arteriolar constriction (4). A further study of the hemodynamic changes involved in the response to angiotonin is the subject of this paper.

\section{METHODS}

In the course of these studies, volunteer, normal convalescent patients from the Third (New York University) Medical Division of Bellevue Hospital were used as subjects. All were at least two hours post prandial and had remained at bed rest for thirty to sixty minutes before observations were begun. One or two cc. of a purified angiotonin solution, for which we are indebted to Dr. Page and his coworkers, were injected intravenously in two subjects while recording arterial pressure from the radial artery. A continuous intravenous infusion, made by adding one volume of angiotonin solution to nine volumes of saline, was given at the rate of three cc. per minute to three subjects, arterial pressure being recorded from the brachial artery.

Cardiac output was determined by a modification of the ballistocardiograph of Starr et al. (5), using the area formula (5). It is recognized that the difficulty of accurate estimation of the cross section of the aorta introduces an error into the calculation of cardiac output, which renders absolute values questionable (6). However, relative values obtained from any one individual are

1 Aided by a grant from the Commonwealth Fund. believed to be valid. All figures for cardiac output and other values determined therefrom (resistance and elasticity coefficient) are subject to revision when this error can be corrected in the light of newer knowledge, and are here presented only in percentile terms. Hamilton's optical manometer (7) was used to measure arterial pressure directly, mean pressure being determined by planimetric measurement of the area under the pulse pressure tracing. We have repeatedly demonstrated that arterial puncture does not cause changes in cardiac output lasting longer than a minute or so. A simultaneous electrocardiogram was recorded with a string galvanometer.

No subjective symptoms other than an occasional complaint of pressure in the head have been noted in the course of these studies. The patients remained at ease throughout all procedures, which were conducted as painlessly as possible.

Having determined cardiac output and mean arterial pressure, peripheral resistance and the efficient elasticity modulus were calculated by equations derived from Frank's $W$ indkesseltheorie. Peripheral resistance, $R$, was expressed in absolute units as dynes $\mathrm{cm}^{-b} \mathrm{sec}$. by the formula, $R=P m \times 1332 / C O$ per second, where $P m$ is mean arterial pressure and $C O$ is cardiac output. The conversion factor of 1332 equals the product of the specific gravity of $\mathrm{Hg}$ and the acceleration of gravity, 980 cm. sec.-

Following Apéria's treatment (8), we have calculated the efficient elasticity modulus $(d P / d V)$, which Apéria indicates by $E_{n}$, from the diastolic pressure function which he called " natural gamma $\left(\gamma_{n}\right)$ ".2 Since $\gamma_{n}=$

\footnotetext{
2 Frank's fundamental equation states that $i=P^{\prime} / E$ $+P / R$, where $i=i(t)$, the stroke flow, and $P=P(t)$, the pressure, at any moment $t, P^{\prime}=P^{\prime}(t)$ is the derivative of the pressure with respect to the time $t, R=$ peripheral resistance, and $E=d P / d V$, the elasticity modulus of the central arterial reservoir, $V$. During diastole in the normal individual, $P^{\prime} / E+P / R=0$, and since $E$ and $R$ are considered constants, $E / R=\gamma$, a constant, (whence $E$ $=\gamma R$ ) the equation now becoming $P^{\prime}+\gamma P=0$, and $\gamma=-P^{\prime}(t) / P(t)$ (i.e., $\gamma=$ the rate of fall of pressure at any moment $t$ ). The gamma value obtained by this equation is in practice not constant throughout diastole and must be replaced by a figure approximately a constant. Apéria points out that this may be done in one of three ways: first, the mean value of gamma, $\gamma_{m}$, may be used; second, gamma may be derived by the use of the method of least squares, giving optimal gamma, $\gamma_{0}$; and last, by determina-
} 


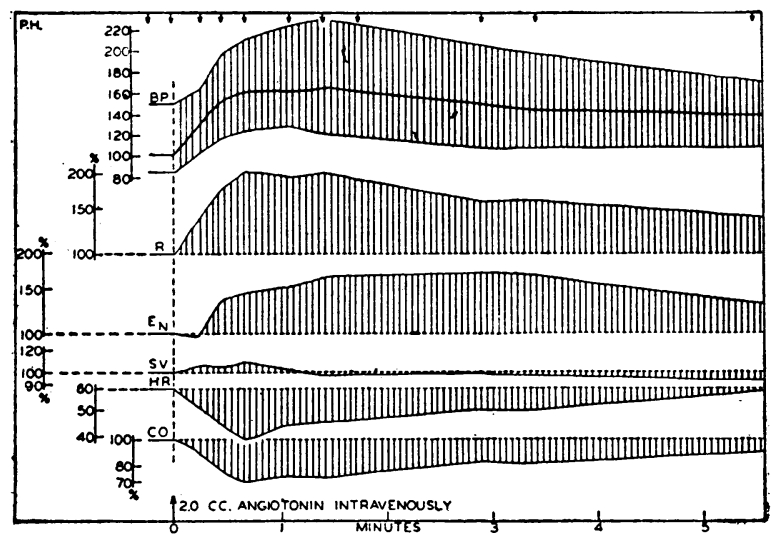

Fig. 1. Hemodynamic Effects of a Single IntraVENOUS INJECTION OF ANGIOTONIN

$P s-P d / D P m d$ (where $P s$ is the systolic pressure, $P d$ the diastolic pressure, $D$ is duration of arterial diastole or of the fall of pressure in the arterial pulse, and $P$ md is the mean pressure during $D$ ), and since $\gamma_{n} R=E_{n}$, as shown below ${ }^{2}, E_{n}=R(P s-P d / D P m d) . E_{n}$ is expressed as dynes $/ \mathrm{cm}^{2}$

We wish to acknowledge our debt to Dr. Seth Hirsh and Mr. Myron Schwartzchild of the Department of Roentgenology of this School for aid and direction in the study of the effect of angiotonin on heart size and pulsation. Kymoroentgenograms and cardiocairograms were obtained before administration of the drug and again at the height of the pressor effect. The cardiocairogram was timed to picture the heart shadow in mid-diastole.

\section{RESULTS}

Figure 1 illustrates the changes which occurred to a greater or lesser degree in all experiments. Peripheral resistance and mean arterial pressure rose consistently, with an increase in pulse pressure. In the experiment illustrated, stroke vol-

tion of natural gamma, $\gamma_{n}$, which is practically most useful. In the determination of $\gamma_{n}$, the diastolic stroke flow (i) is zero:

$\int_{S}^{T} i(t) d t=\int_{S}^{T}\left(\frac{P^{\prime}}{E}+\frac{P}{R}\right) d t=\int_{S}^{T}\left(P^{\prime}+\gamma_{n} P\right) d t=0$,

where $S$ is time of systole and $T$ is the end of the pulse period.

$$
\therefore \gamma_{n}=-\frac{\int_{S}^{T}\left(P^{\prime}\right) d t}{\int_{S}^{T} P d t}=\frac{P_{s}-P d}{\int_{S}^{T} P d t},
$$

where $P_{s}$ is systolic pressure, $P_{d}$ is diastolic pressure, $\int_{S}^{T} P d t$ is the product of mean pressure during fall of pressure from systolic peak to end of diastole and duration of this fall of pressure.

Thus it can be seen that $E_{n}=\gamma_{n} R$. ume decreased-though this was not invariably the case, some subjects showing virtually no change -and in no instance did stroke volume increase.

$B P$, blood pressure (Hamilton manoneter) in $\mathrm{mm}$. $\mathrm{Hg} ; R$, peripheral resistance ; $E_{n}$, efficient elasticity modulus; $S V$, stroke volume; $H R$, heart rate; $C O$, cardiac output. The arrows at the top indicate points at which the ballistocardiographic and manometric records were recorded or read.

Because of uncertainties in the absolute values of the stroke volume, as determined by the ballistocardiograph, this measurement and all dependent measurements ( $C O$, $E_{n}$, and $R$ ) are expressed as percentile changes relative to the respective control values.

In four single injection experiments, cardiac output was substantially reduced, the reduction being the result of decreased heart rate, which was invariable, and such reduction in stroke volume as may have been present. With a single exception (which is shown in Figure 3), $E_{n}$ increased markedly, indicating decreased distensibility of the central arterial reservoir.

Figure 2 illustrates the effect of the continuous infusion of angiotonin (three experiments). The hemodynamic changes were similar to those induced by a single injection, except that bradycardia is not evident and the decrease in cardiac output is rather less.

$\mathrm{X}$-ray studies of the heart shadow in four additional subjects following intravenous injection of angiotonin revealed no consistent changes, two subjects showing a slight increase in all diameters, one showing no change, and one showing a slight

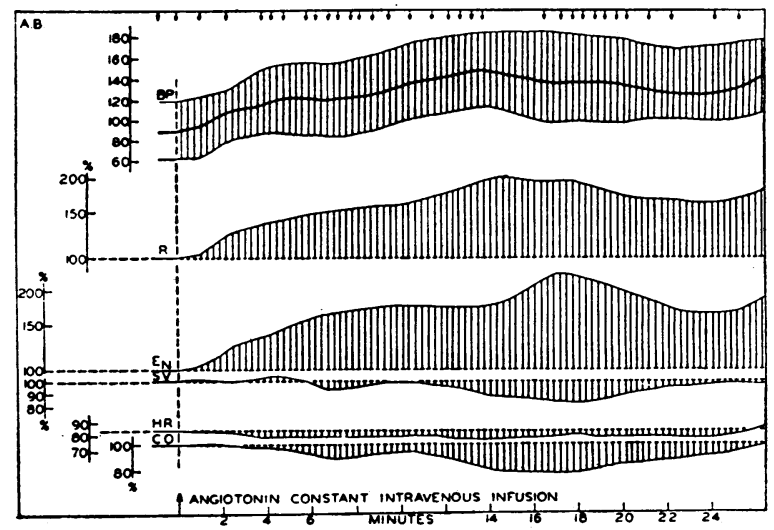

Fig. 2. Hemodynamic Effects of the Continuous INTRAVENOUS INFUSION OF ANGIOTONIN

Symbols same as in Figure 1. 


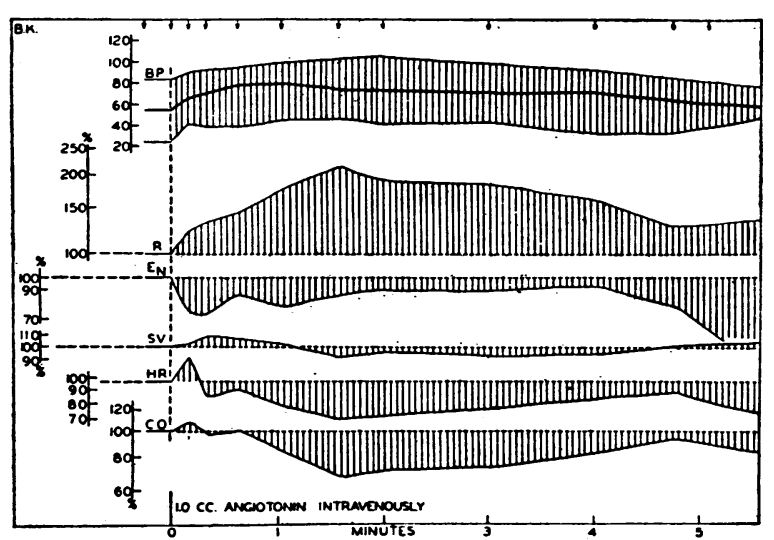

Fig. 3. An Anomalous Instance Where $E_{n}$ Was Apparently Decreased. After Angiotonin

A possible explanation is discussed in the text.

decrease in all diameters. However, kymoroentgenograms showed a consistent decrease in amplitude of ventricular waves, which was marked in three cases, amounting to as much as $\mathbf{5 0}$ per cent. This apparently indicates a decreased emptying of the heart and, since the diastolic volume was not appreciably increased, a decrease in stroke volume. This qualitatively confirms our observations with the ballistocardiograph. There was little change in amplitude of auricular waves.

An interesting change in pulse pressure contour, consisting of the development of a secondary pre-dicrotic wavelet which was apparently a reflected wave, made its appearance approximately ten seconds after injection of the drug. This change was often associated with sudden, marked slowing and irregularity of the pulse.

No change in the electrocardiogram was observed.

\section{DISCUSSION}

Our data show that in man angiotonin is a definitely pressor substance, producing an elevation in systolic, diastolic and mean pressure, with a widening of pulse pressure. The increase in blood pressure appears to arise out of the increased peripheral resistance, presumably occasioned by arteriolar constriction in as yet undetermined sites.

The efficient elasticity modulus $\left(E_{n}\right)$ is found to increase in every case save one (Figure 3 ), which will be discussed below. This indicates that the distensibility of the central arterial reser- voir decreases following the administration of angiotonin. It is, however, uncertain what factors operate to bring about this change. It may be that angiotonin acts upon the smooth musculature of the central arteries in the same manner that it acts upon the arterioles, increasing the rigidity of these arteries and thereby decreasing their distensibility. It is, however, well known that the distensibility of arteries decreases with increasing distention, and it may be expected that, when the resistance to outflow from the central arteries is increased by vasoconstriction, the cardiac output remaining relatively constant, increased distention of these arteries will occur and will be reflected in an increase in $E_{n}$. It is difficult at the present time to differentiate between these alternative explanations. It may be noted that if angiotonin acts directly on the central arteries to decrease their distensibility (in the sense of active contraction), we would expect their distensibility to be reduced more than would be the case if increased filling were the only factor. Comparison of our data with the curves published by Bramwell, McDowall and McSwiney (9) for muscular arteries reveals that the decrease in distensibility in our data is less than is to be expected from the equivalent changes in diastolic pressure. This fact might be interpreted as indicating that the distensibility-volume relations are different in the central arterial reservoir and the muscular arteries studied by Bramwell, McDowall and McSwiney, in which view any direct action of angiotonin on these arteries must be slight. We cannot, moreover, rule out a reflex mechanism, such as that observed in the dog by Dow and Hamilton (10), which on elevation of blood pressure increases the distensibility of the aorta. In any case the increase in pulse pressure observed in all subjects, and illustrated in Figures 1 and 2, is entirely the result of the change in distensibility of the central arterial reservoir, whatever its origin, and not the result of an increase in stroke volume.

We have mentioned that on one occasion (Figure 3) $E_{n}$ fell following administration of one cc. of angiotonin intravenously. Two cc. in the same individual caused an elevation of $E_{n}$. It does not seem likely that distensibility of the central arterial reservoir increased in the face of disten- 
tion in this reservoir, as revealed by a slight elevation of diastolic pressure; it is more likely that this case emphasizes the desirability of caution in interpreting mathematical analysis of the physiological forces which are involved. The calculated values involve several assumptions and yield what at best are only approximations which are useful for showing directional changes and indicating the magnitude of these changes. For example, the determination of $\gamma_{n}$ is apparently valid only in pulse pressure curves which show a slight-tomoderate degree of dicrotism, since the occurrence of a marked dicrotic notch causes an apparent decrease in $P$ md, thus elevating $E_{n}$. With the exception of the subject illustrated in Figure 3, the pulse pressure curves analyzed here have shown only a slight dicrotism, but in that subject dicrotism occurred to a marked degree during the control period, associated with marked hypotension. Consequently, the control values of $E_{n}$ are relatively high, and fall abruptly to a lower level after angiotonin, when dicrotism is reduced. Thus the change in pulse form (degree of dicrotism) may explain the apparent drop in $E_{n}$ following the administration of angiotonin. Had excessive dicrotism not been present in the control periods, the control value of $E_{n}$ might have been much lower and the changes following angiotonin might have resembled those observed in the other subjects.

The validity of $E_{n}$ as an index of elasticity changes is supported by the fact that conversion of this value to pulse wave velocity, using Bramwell and Hill's (11) equation where pulse wave velocity $=3.57 / \sqrt{\text { per centile distensibility, }}$ gives velocities similar to those found in the literature $(12,13)$ for the central arteries. In addition, Apéria (8) has shown that $E_{n}$ increases with age, an expected physiological result not obtainable by other indirect measurements of elasticity. Further studies of the relation between pulse wave velocity and $E_{n}$ are in progress.

Bradycardia has in nearly every case been the chief cause for the reduction in cardiac output; presumably, the bradycardia represents vagal slowing, elicited through the carotid sinus and aortic depressor reflexes. Where the pressure rises slowly (Figure 2) no bradycardia ensues. In no instance did stroke volume increase, a rather surprising result since Wilkins (personal communi- cation) has found a marked increase in cubital venous pressure, and Cournand and Ranges (unpublished data) have found a marked rise in right auricular pressure, after angiotonin administration. Since $x$-ray studies fail to show cardiac dilatation, it is inferred that the failure of the heart to increase its stroke volume in the face of increased venous pressure is attributable to increased "cardiac tone." This conclusion is further supported by the demonstration of decreased diastolic size of the isolated mammalian heart in response to angiotonin (3). ${ }^{\mathrm{s}}$

\section{SUM MARY}

1. Cardiac output, mean arterial pressure, peripheral resistance and efficient elasticity modulus have been determined following intravenous administration of angiotonin.

2. In all experiments, mean arterial pressure and peripheral resistance rose sharply. The pulse pressure tended to widen. With one exception, the efficient elasticity modulus rose sharply. Cardiac output fell as a result of a marked bradycardia. There was little change in stroke volume and, where a change occurred, it was in the direction of a decrease. Response to small single injections and to continuous intravenous infusions differed only in the absence of bradycardia in the latter.

3. Kymoroentgenogram and cardiocairogram studies revealed little change in heart size. However, there was present a consistent decrease in amplitude of ventricular waves.

4. It is concluded that angiotonin acts directly upon the musculature of the cardiovascular system, producing arteriolar vasoconstriction and possibly increased "cardiac tone." Whether the distensibility of the central arterial reservoir is specifically decreased by angiotonin, or whether the observed change is attributable simply to increased distention of the central arteries, cannot be answered with certainty from the present data.

We wish to acknowledge our indebtedness to Miss Betty J. Crawford for her aid in computations.

8 Wilkins and Duncan (J. Clin. Invest., 1941, 20, 442) have shown that angiotonin decreases the vital capacity. The above discussion should perhaps be qualified in the light of this fact. Concerning cardiac tone see Bibliography (14). 


\section{BIBLIOGRAPHY}

1. Page, I. H., and Helmer, O. M., A crystalline pressor substance (angiotonin) resulting from the reaction between renin and renin-activator. J. Exper. Med., 1940, 71, 29.

2. Page, I. H., The nature of clinical and experimental hypertension. J. Mt. Sinai Hosp., 1941, 8, 3.

3. Lorber, V., and Visscher, M. B., The action of angiotonin on the completely isolated mammalian heart. Proc. Am. Physiol. Soc., 1941, p. 177.

4. Corcoran, A. C., Kahlstaedt, K. G., and Page, I. H., Changes of arterial blood pressure and renal hemodynamics by injection of angiotonin in human beings. Proc. Soc. Exper. Biol. and Med., 1941, 46, 244.

5. Starr, I., and others, Studies on the estimation of cardiac output in man, and of abnormalities in cardiac function, from the heart's recoil and the blood's impacts, the ballistocardiogram. Am. J. Physiol., 1939, 127, 1.

6. Cournand, A., and Ranges, H. A., Determination of cardiac output in man by direct Fick method and the ballistocardiograph. Proc. Am. Physiol. Soc., 1941, p. 63.

7. Hamilton, W. F., Brewer, G., and Brotman, I., Pres- sure pulse contours in the intact animal. I. Analytical description of a new high frequency hypodermic manometer with illustrative curves of simultaneous arterial and intracardiac pressures. Am. J. Physiol., 1934, 107, 427.

8. Apéria, A., Hemodynamical studies. Skand. Arch. f. Physiol., 1940, Suppl. 16, 83, 1.

9. Bramwell, J. C., McDowall, R. J. S., and McSwiney, B. A., The variation of arterial elasticity with blood pressure in man (Part I). Proc. Roy. Soc. London, Ser. B., 1922, 94, 450.

10. Dow, P., and Hamilton, W. F., An experimental study of the velocity of the pulse wave propagated through the aorta. Am. J. Physiol., 1939, 125,60 .

11. Bramwell, J. C., and Hill, A. V., The velocity of the pulse wave in man. Proc. Roy. Soc. London, Ser. B., 1922, 93, 298.

12. Bazett, H. C., and Dreyer, N. B., Measurements of pulse wave velocity. Am. J. Physiol., 1922, 63, 94.

13. Haynes, F. W., Ellis, L. B., and Weiss, S., Pulse wave velocity and arterial elasticity in arterial hypertension, arteriosclerosis, and related conditions. Am. Heart J., 1936, 11, 385.

14. Johnson, V., and Katz, L. N., Tone in the mammalian ventricle. Am. J. Physiol., 1937, 118, 26. 